\title{
Avaliação físico-química e nutricional de farinhas de banana verde com casca elaboradas a partir de variedades distintas
}

\section{Physical, chemical and nutritional evaluation of flours prepared with pulp and peel of green banana from different varieties}

\author{
Arianny Amorim de Sá1* (1), Maria Isabel Almeida Gonçalves², \\ Thayris Rodrigues Vasconcelos ${ }^{2}$, Marianne Louise Marinho Mendes ${ }^{3}$,
} Cristhiane Maria Bazílio de Omena Messias ${ }^{3}$

${ }^{1}$ Universidade de Pernambuco (UPE), Programa de Pós Graduação em Ciência e Tecnologia Ambiental (PPGCTA), Petrolina/PE - Brasil

${ }^{2}$ Universidade de Pernambuco (UPE), Departamento de Nutrição, Petrolina/PE - Brasil

3Universidade de Pernambuco (UPE), Departamento de Nutrição e Programa de Pós Graduação em Ciência e

Tecnologia Ambiental (PPGCTA), Petrolina/PE - Brasil

*Corresponding author: Arianny Amorim de Sá, Universidade de Pernambuco (UPE), Campus Petrolina, Curso de Nutrição, BR 203 km 2 s/n, Campus Universitário, Vila Eduardo, CEP: 56328 - 903, Petrolina/PE, e-mail: ariannynutricionista@gmail.com

Cite as: Sá, A. A., Gonçalves, M. I. A., Vasconcelos, T. R., Mendes, M. L. M., Messias, C. M. B. O. (2021).

\begin{abstract}
Resumo
O processamento de frutos ainda verdes para produção de farinhas pode ser considerado uma opção para minimizar as perdas pós-colheita e agregar valor ao produto. O objetivo do trabalho foi avaliar a composição físicoquímica e nutricional de farinhas de banana verde (FBV) com casca, obtidas a partir de diferentes variedades. Para a produção das farinhas, foram utilizadas: banana-prata, banana-da-terra e banana-maçã. A secagem foi realizada em estufa a $105^{\circ} \mathrm{C}$, por 12 horas. Foram realizadas análises de umidade, pH, cinzas, carboidratos, proteínas, lipídios, fibras e amido. Todas as farinhas produzidas apresentaram teor de umidade adequado. Quanto ao teor de fibras, todas as variedades apresentaram bons resultados, o que se deve ao fato de preservar a casca na sua produção, bem como um teor de amido elevado para banana-da-terra e banana-maçã, o que reforça a grande quantidade no fruto ainda verde. Todas as farinhas apresentaram um baixo valor calórico. Portanto, é viável a utilização da FBV com casca de todas as variedades estudadas, principalmente pelo teor de fibras, validando, assim, o presente trabalho. É possível destacar ainda a importância da produção de farinha de banana verde com casca, visando manter um maior número de nutrientes, por vezes encontrados na casca, bem como possibilitar o aproveitamento integral da banana.
\end{abstract}

Palavras-chave: Alimento funcional; Amido; Desperdício; Valor nutricional; Umidade; Carboidratos.

\section{Abstract}

Processing of green fruits for flour production can be considered an option to minimize post-harvest losses and add value to the product. This study aimed to evaluate the physical, chemical and nutritional composition of flours 
prepared with pulp and peel of green banana from different varieties. Silver bananas, plantains, and apple bananas were used to produce the flours. Drying was carried out in an oven at $105^{\circ} \mathrm{C}$ for 12 hours. Analyses of $\mathrm{pH}$, moisture, ash, carbohydrate, protein, lipid, fiber and starch contents were performed. All the flours produced showed adequate moisture content. As for fiber content, the flours prepared with all banana varieties presented good results, which was due to the fact that the peel was preserve in their production. High starch content was observed for plantains and apple bananas, which reinforces the large amount of starch found in the green fruit. All flours had low caloric value. The results showed the feasibility of using green banana flour ) prepared with pulp and peel of these varieties mainly because of the fiber content, thus validating the present study. It is also worth mentioning the importance of producing green banana flour with peel, aiming to maintain a greater amount of nutrients, sometimes found in the peel, as well as enabling the full use of the fruit.

Keywords: Functional food; Starch; Waste; Nutritional value; Moisture; Carbohydrates.

\section{Introdução}

A banana (Musa spp.) é uma fruta tropical amplamente consumida no mundo (Souza et al., 2011). Pertencente à família Musaceae, apresenta cerca de 30 espécies conhecidas do gênero Musa e mais de 700 variedades (Asmar et al., 2013). Mundialmente, a banana é a fruta mais produzida, com 17,4\% do volume de produção de frutas, e, no panorama nacional, ocupa a segunda posição, respondendo por 16,7\% do volume de frutas, perdendo apenas para a produção de laranja (Secretaria de Agricultura e do Abastecimento, 2016). Além disso, a facilidade de propagação e o manejo fazem com que essa fruta seja uma das mais comercializadas mundialmente, apresentando relevância econômica e social, principalmente, nas regiões tropicais (Coelho Junior, 2013).

O Brasil, em 2013, produziu aproximadamente 7 milhões de toneladas, sendo a região Nordeste a principal produtora, com $\sim 37 \%$, seguida da Sudeste, com $\sim 32 \%$ da produção (Instituto Brasileiro de Geografia e Estatítica, 2017). Em 2011, o consumo médio da fruta foi na ordem de $31 \mathrm{~kg}$ por habitante ao ano (Food and Agriculture Organization of the United Nations, 2011).

Em relação à sua importância econômica, tolerância a doenças e resistência ao frio, as seguintes variedades se destacam: prata, pacová, prata anã, maçã, terra e nanica (Mota et al., 2000).

A melhor aceitação da banana madura se deve aos seus aspectos sensoriais e nutricionais, consistindo em fonte energética por causa da presença de carboidratos e de minerais importantes, como potássio e vitaminas (Matsuura et al., 2004). O fruto verde tem despertado interesse do mercado consumidor, pois, além do seu valor nutricional, com quantidades consideráveis de vitaminas $\mathrm{B}$ e $\mathrm{C}$ e de sais minerais como potássio e cálcio (Leonel et al., 2011), possui amido resistente (Tribess et al., 2009), fitoesteróis, compostos fenólicos (Haslinda et al., 2009) e capacidade antioxidante (Wang et al., 2012).

Entretanto, no estágio de maturação verde, a banana não é muito consumida, principalmente em razão da típica dureza e sua elevada adstringência, acarretada pela presença de compostos fenólicos solúveis, como os taninos (Sarawong et al., 2014; Andrade et al., 2018). Essa adstringência é reduzida durante o processo de amadurecimento, quando ocorre a polimerização dessas substâncias e a degradação do amido, levando ao aumento da doçura e da maciez e à redução da acidez, característicos da fruta madura. Consequentemente, a obtenção de farinhas é a principal alternativa para assegurar a utilização dos frutos verdes pela indústria de alimentos (Sarawong et al., 2014).

A farinha de banana verde (FBV) é uma rica fonte de potássio, fósforo, magnésio, cobre, manganês e zinco, quando comparada aos demais tipos de farinhas existentes no mercado (Borges et al., 2009). Suas fibras solúveis e insolúveis desempenham diversas funções no organismo, como a regulação da função intestinal, no qual atua retardando o esvaziamento gástrico, além de auxiliar na redução dos níveis de colesterol no sangue e de ser utilizado como substrato para fermentação por bactérias aeróbicas do cólon (Sardá, 2015; Waszak \& Ferreira, 2011). 
Segundo Vernaza et al. (2011), a produção de FBV encontra ampla aplicação na indústria de alimentos, sobretudo na elaboração de produtos de panificação, produtos dietéticos e alimentos infantis, sendo uma fonte de amido resistente e sais minerais. Além dos benefícios nutricionais, a produção de FBV contribui para a redução das perdas pós-colheita, aumento do tempo de vida de prateleira e agregação de valor à fruta (Bezerra et al., 2013; Castelo-Branco et al., 2017).

Dessa forma, o presente trabalho tem como objetivo avaliar a composição físico-química e nutricional de farinhas de banana verde com casca, obtidas a partir de diferentes variedades, visando ao aproveitamento integral da matéria-prima.

\section{Material e métodos}

Para a obtenção da FBV, foram utilizadas três variedades de bananas comercializadas na região do município de Petrolina/PE, onde foram coletadas no período de agosto a outubro de 2018: os cultivares prata (BP), terra (BT) e maçã (BM). A elaboração da farinha foi realizada no Laboratório de Técnica Dietética e no Laboratório de Bromatologia da Universidade de Pernambuco, campus de Petrolina/PE.

\subsection{Elaboração das farinhas}

As bananas foram adquiridas com o grau de maturação totalmente verdes e verdes com traços amarelos, seguindo os parâmetros da escala de Von Loesecke (Programa Brasileiro para a Modernização da Horticultura \& Produção Integrada de Frutas, 2006). Foram descartadas as bananas que apresentavam injúrias ou danos físicos.

Para a produção das farinhas, foi utilizada a metodologia proposta por Borges et al. (2009). Inicialmente, foi retirada a parte superior que faz a junção das bananas - engaço e almofada -, aproveitando todo o restante de forma integral.

Em seguida, foram submetidas à lavagem em água corrente para a retirada de sujidades. Posteriormente, foram higienizadas com hipoclorito de sódio $15 \mathrm{ppm}$ por 15 minutos. A água foi descartada, e o processo de higienização com o hipoclorito foi repetido por mais 10 minutos em mais duas operações, visando reduzir a carga microbiana da casca.

Posteriormente, foram cortadas em rodelas em uma espessura de $3 \mathrm{~mm}$, com o auxílio de um Mandolin (fatiador manual de frutas e legumes), mantendo a casca da fruta e demais partes, aproveitando a banana integralmente.

As bananas, devidamente cortadas em rodelas, foram submetidas a uma nova solução, contendo $5 \mathrm{~g} / \mathrm{L}$ do ácido cítrico, evitando, assim, o escurecimento enzimático das bananas, e permaneceram submersas por 10 minutos. Posteriormente, as rodelas de cada variedade de banana seguiram para a estufa com circulação de ar forçado a $105^{\circ} \mathrm{C}$ por 12 horas.

Após a secagem, foram trituradas em miniprocessador HC31BD (Becker\&Decker). A etapa de trituração foi realizada múltiplas vezes, até processar ao máximo o material, visando a um maior rendimento da farinha. Em seguida, a farinha foi peneirada em peneira de aço inox, sendo a operação repetida até obter uma cor clara e uniforme.

Posteriormente, a farinha foi pesada para se obter o cálculo do rendimento final. Em seguida, ela foi acondicionada em potes plásticos, identificados de acordo com a variedade e envolvidos por papel alumínio, objetivando uma melhor conservação e durabilidade. As farinhas foram armazenadas à temperatura ambiente $\left(25^{\circ} \mathrm{C}\right)$. 


\subsection{Cálculo do rendimento}

Para o cálculo do rendimento das farinhas produzidas, foi utilizada a fórmula a seguir (Equação 1), com base na metodologia proposta por Menezes et al. (2009).

$$
\begin{aligned}
& \% \mathbf{R}=\mathbf{P F} \times \mathbf{1 0 0} / \mathbf{P I} \\
& \mathrm{R}=\text { porcentagem de rendimento da farinha; } \\
& \mathrm{PF}=\text { peso final (massa da farinha após a secagem); } \\
& \mathrm{PI}=\text { peso inicial (massa da banana antes de secagem). }
\end{aligned}
$$

\subsection{Caracterização físico-química das farinhas}

Ao final de todo o processo, as farinhas das três variedades de bananas passaram por análises, sendo utilizada como referência a metodologia para análises físico-químicas em alimentos, de Adolfo Lutz (Instituto Adolfo Lutz, 2008). A determinação de umidade foi realizada por meio do método de perda por dessecação $(012 / \mathrm{IV})$, mediante secagem direta em estufa a $105{ }^{\circ} \mathrm{C}$. A análise de $\mathrm{pH}$ foi realizada por processo eletrométrico com a leitura do potencial hidrogeniônico em pHmetro (017/IV) (Instituto Adolfo Lutz, 2008).

Posteriormente, foram realizadas as análises de quantificação de proteínas totais pelo método de Kjeldahl clássico (036/IV), de lipídios por extração direta em Soxhlet (032/IV), de cinzas por meio de resíduo por incineração(018/IV), de amido (043/IV) e de fibra bruta (044/IV) (Instituto Adolfo Lutz, 2008).

A quantificação de carboidratos foi realizada pelo método de diferença do valor de 100 , subtraído do somatório dos valores já obtidos de umidade, cinzas, proteínas e lipídios (Mendes-Filho et al., 2014), como demonstra a equação a seguir (Equação 2):

Carboidratos $(\%)=100-(\%$ umidade $+\%$ cinzas $+\%$ lipídios $+\%$ proteínas $)$

Por fim, a determinação do valor energético total foi expresso por meio dos resultados obtidos dos macronutrientes: carboidratos $(\mathrm{C})$, proteínas $(\mathrm{P})$ e lipídios (L), utilizando-se da equação (Equação 3) que expressa o valor de $\mathrm{kcal} / 100 \mathrm{~g}$.

Valor energético $($ kcal $/ 100 g)=($ Cx4 $)+($ Px4 $)+($ Lx9 $)$

Em que:

$\mathrm{C}=$ valor de carboidrato $(\%)$;

$\mathrm{P}=$ valor de proteína $(\%)$;

$\mathrm{L}=$ valor de lipídio (\%);

4 = fator de conversão em kcal determinado em bomba calorimétrica para proteína e carboidrato;

9 = fator de conversão em kcal determinado em bomba calorimétrica para lipídio.

\subsection{Análise dos dados}

Os dados das análises foram tabelados no programa Microsoft Excel 2010 e submetidos ao programa Bioestat 5.0. Foram aplicados o teste de variância ANOVA (1 critério), para comparar as médias, e o teste de Tukey, para identificar grupos que diferem entre si. Foi adotado um nível de significância de 5\% $(p<0,05)$.

\section{Resultados e discussão}

O rendimento final das farinhas das três variedades de banana segue apresentado no Tabela 1. 
Tabela 1. Rendimento das farinhas de bananas, Petrolina/PE, 2020.

\begin{tabular}{cc}
\hline Farinhas & Rendimento (\%) \\
\hline Farinha de banana-prata & $21 \%$ \\
\hline Farinha de banana-da-terra & $27 \%$ \\
\hline Farinha de banana-maçã & $24 \%$ \\
\hline
\end{tabular}

Pires et al. (2014), ao avaliarem a FBV da variedade pacová, observaram valores de rendimento entre 10,11 e $29,29 \%$, o que foi atribuído à eficiência do processo de secagem. Assim, quanto mais a amostra fica dura e quebradiça, mais facilita a trituração e peneiramento, aumentando o volume final. Castro (2019) obteve $14 \%$ de rendimento ao produzir FBV, valor abaixo do encontrado no atual estudo, o que pode ser evidenciado por causa do fato de preservar a casca, aumentando, dessa forma, o rendimento.

Andrade et al. (2018) analisaram FBV de dois tipos de variedades - banana-prata e banana-caturra -, observando maior rendimento para a banana-prata (26,4\%) quando comparada ao presente estudo (21\%); porém, foi verificado um rendimento menor referente à FBV da variedade caturra $(18,9 \%)$, ao ser comparada com todas as amostras do presente estudo. Essas diferenças podem ser atribuídas às diferentes metodologias, principalmente tempo e temperatura, aplicadas na produção de FBV.

Entre as três variedades de FBV com casca analisada, os resultados foram expressos em base seca, bem como o valor calórico das farinhas, dispostos na Tabela 2.

Tabela 2. Composição físico-química e valor calórico das farinhas de banana verde com casca em base seca, Petrolina/PE, 2020.

\begin{tabular}{cccc}
\hline & & & Farinhas \\
Composição centesimal & FBVP* & FBVT $^{*}$ & FBVM $^{*}$ \\
\cline { 2 - 4 } & $6,01^{\mathrm{a}} \pm 1,32$ & $8,84^{\mathrm{a}} \pm 1,44$ & $7,59^{\mathrm{a}} \pm 1,70$ \\
\hline Umidade (\%) & $5,55^{\mathrm{a}} \pm 0,16$ & $5,26^{\mathrm{a}, \mathrm{b}} \pm 0,15$ & $4,78^{\mathrm{a}, \mathrm{c}} \pm 0,09$ \\
\hline $\mathrm{pH}$ & $80,86^{\mathrm{a}} \pm 0,52$ & $86,55^{\mathrm{b}} \pm 0,27$ & $84,40^{\mathrm{c}} \pm 0,03$ \\
\hline Carboidratos $(\mathrm{g} / 100 \mathrm{~g})$ & $5,40^{\mathrm{a}} \pm 0,24$ & $2,40^{\mathrm{b}} \pm 0,27$ & $3,40^{\mathrm{c}} \pm 0,22$ \\
\hline Proteínas totais $(\mathrm{g} / 100 \mathrm{~g})$ & $0,97^{\mathrm{a}} \pm 0,03$ & $0,98^{\mathrm{a}} \pm 0,01$ & $1,09^{\mathrm{a}} \pm 0,01$ \\
\hline Lipídios totais $(\mathrm{g} / 100 \mathrm{~g})$ & $4,19^{\mathrm{a}} \pm 0,11$ & $3,09^{\mathrm{b}} \pm 0,02$ & $3,84^{\mathrm{c}} \pm 0,01$ \\
\hline Cinzas $(\mathrm{g} / 100 \mathrm{~g})$ & $67,20^{\mathrm{a}} \pm 0,80$ & $73,25^{\mathrm{b}} \pm 2,82$ & $73,90^{\mathrm{b}} \pm 1,30$ \\
\hline Amido $(\mathrm{g} / 100 \mathrm{~g})$ & $7,25^{\mathrm{a}} \pm 0,08$ & $6,29^{\mathrm{b}} \pm 0,08$ & $8,44^{\mathrm{c}} \pm 0,31$ \\
\hline Fibra bruta $(\mathrm{g} / 100 \mathrm{~g})$ & 332,62 & 332,90 & 333,6 \\
\hline Calorias $(\mathrm{kcal} / 100 \mathrm{~g})$ & &
\end{tabular}

FBVP*: Banana-prata, FBVT*: Banana-da-terra, FBVM*: Banana-maçã. Os resultados estão expressos na forma de média \pm desvio-padrão. Médias na mesma linha com letras diferentes são estatisticamente significantes $(p<0,05)$.

Segundo o regulamento técnico para farinhas, amido de cereais e farelos, estabelecido pela $\mathrm{RDC} \mathrm{n}^{\circ} 263$, de 22 de setembro de 2005 (Agência Nacional de Vigilância Sanitária, 2005), a umidade máxima permitida é de até $15 \%(\mathrm{~g} / 100 \mathrm{~g})$, estando de acordo com os dados encontrados neste estudo para FBVP $(6,01 \pm 1,32)$, FBVT $(8,84 \pm 1,44)$ e FBVM $(7,59 \pm 1,70)$.

Melo (2014) encontraram valores semelhantes para umidade em FBV da variedade prata $(6,3 \%)$, corroborando o valor obtido no presente estudo. Gonçalves et al. (2017) encontram valores superiores de umidade para FBV da casca in natura $(14,54 \%)$ e da polpa $(15,05 \%)$ realizados em bananas do subgrupo 
cavendish. A baixa umidade pode ser explicada em razão da preservação da casca. Nesse sentido, a umidade é um aspecto importante para a avaliação da vida de prateleira das farinhas, visto que quantidades elevadas de umidade podem reduzir sua estabilidade e favorecer a sua deterioração.

Ao analisar a FBVP, os resultados indicaram um maior teor de proteínas e fibras quando comparada à FBVT. Em contrapartida, a FBVT apresentou valores de amido superiores quando equiparada à FBVP (Tabela 1). Os dados referentes a proteínas, fibras e amido apresentaram significância estatística $(p<0,05)$. Nascimento et al. (2017), ao estudarem a farinha da banana-da-terra, encontraram um teor de amido total de $66,8 \%$, relativamente mais baixo que o encontrado no presente estudo $(73,25 \%)$ para a mesma variedade.

O teor de cinzas obteve significância estatística quando comparada à FBVP e FBVT. Bezerra et al. (2013), em estudo anterior realizado com bananas do cultivar cavendish, relataram teores de proteína de 4,33 g/100 g, cinzas de 2,72 g/100 g, fibras de 15,52 g/100 g e amido de 68,42 g/100 g. Essa variação de teores pode ser explicada por causa da grande variedade de cultivares de bananas encontradas no Brasil, bem como as diferentes condições de plantio.

A FBVM apresentou um maior teor de fibras e menor $\mathrm{pH}$ quando comparada à FBVT. Em estudo realizado com FBV do tipo maçã, mantendo a casca, foi verificado um $\mathrm{pH}$ mais ácido $(5,70)$ em comparação à farinha sem a casca da banana $(6,05)$ (Almeida et al., 2017). Savlak et al. (2016) também encontraram valores de pH mais ácido $(5,65)$ como no presente estudo. Nesse sentido, a determinação do $\mathrm{pH}$ fornece dados valiosos na apreciação do estado de conservação de alimentos e possibilita um maior controle na elaboração das farinhas (Gonçalves et al.,2017).

A produção de FBV com a preservação da casca da fruta pode ser uma alternativa para agregar valor nutricional às refeições, principalmente pelo teor significativo de fibras encontrado, como no estudo realizado por Bezerra et al. (2013), corroborando, dessa forma, os resultados da pesquisa em questão, ao explanar dados estatisticamente significantes para o teor de fibras em todas as variedades avaliadas. Bertolini et al. (2010) também verificaram, em farinhas que mantiveram a casca, maiores teores de cinza e fitoesteróis, sendo o último nutriente de grande importância para a qualidade da alimentação dos indivíduos (De Melo et al., 2019).

Em relação ao teor de carboidratos, em estudos realizados por Borges et al. (2009) com o cultivar prata no estágio verde, foi verificado um teor de $87,92 \mathrm{~g} / 100 \mathrm{~g}$, enquanto, no presente estudo, a quantidade de carboidrato foi um pouco menor para a mesma variedade, podendo ser explicado pela multiplicidade de metodologias existentes para as análises, bem como as condições climáticas e de plantio para cada localidade, o que pode interferir em sua composição centesimal. Ainda, Borges et al. (2009) e Juarez-Garcia et al. (2006), ao avaliarem a FBV, obtiveram, respectivamente, dados de amido no valor de 72,72 g/100 g e $73,4 \mathrm{~g} / 100 \mathrm{~g}$, sendo compatíveis com o encontrado neste estudo, referente à FBVT $(73,25 \pm 2,82)$ e FBVM $(73,90 \pm 1,30)$, reforçando, assim, os resultados de alto teor de amido que compõe a banana em estágio de maturação verde (Gonçalves et al., 2017).

É importante salientar que boa parte do amido presente na banana verde é representado pela fração do amido resistente, que tem benefícios importantes à saúde humana, conforme identificado em estudo realizado por Ramos et al. (2010), que obtiveram valores de amido resistente para variedade prata de 20,43 g/100 g e para a variedade maçã de $20,74 \mathrm{~g} / 100 \mathrm{~g}$, o que pode indicar também uma quantidade expressiva do amido resistente no presente estudo. Vale ressaltar que o amido pode ter sofrido uma redução importante por causa do processo de secagem empregado, o que pode ter ocasionado a sua gelatinização.

Foram constatados valores calóricos para FBVP, FBVT e FBVM, respectivamente de 332,62 kcal, $332,90 \mathrm{kcal}$ e $333,6 \mathrm{kcal}$, os quais representam valores baixos quando comparados a pesquisas anteriores, que obtiveram valores de $373 \mathrm{kcal}$ a $385 \mathrm{kcal}$ (Borges et al., 2009), o que incrementa, de fato, pontos positivos para o presente estudo, já que as farinhas elaboradas oferecem benefícios à saúde com um menor valor calórico total, podendo ser incluída em um contexto alimentar saudável. 
Por fim, é importante destacar que a banana é uma das frutas mais desperdiçadas no mundo. Um estudo realizado por Mattsson et al. (2018) constatou que a banana faz parte de um grupo de sete variedades de vegetais e frutas que mais são desperdiçadas, sendo a primeira desse ranking, enfatizando a importância de utilizá-la ainda verde e com casca para produção de farinhas, que podem ser acrescentadas a vários produtos alimentícios, enriquecendo-os.

\section{CONCLUSÃO}

Em virtude dos aspectos analisados, é viável a utilização das farinhas de banana verde com casca de todas as variedades estudadas, principalmente pelo teor de fibras encontrado, validando, assim, o presente trabalho. É importante ressaltar que a FBVM se destacou com as maiores quantidades de fibras e amido, nutrientes importantes na elaboração de futuros produtos funcionais.

É preciso destacar ainda a importância da produção das farinhas de banana verde com casca, visando manter um maior número de nutrientes, por vezes encontrados na casca, bem como possibilitar o aproveitamento integral da banana, que é extremamente perecível, contribuindo não apenas para a saúde da população, mas também para um planeta mais sustentável.

\section{AGRADECIMENTOS}

À Universidade de Pernambuco, campus de Petrolina/PE; ao Programa Institucional para Excelência na Qualidade do Stricto Sensu - Apoio ao Pesquisador (APQ); ao Conselho Nacional de Desenvolvimento Científico e Tecnológico (CNPq); à Fundação de Amparo à Ciência e Tecnologia de Pernambuco (FACEPE); e à Coordenação de Aperfeiçoamento de Pessoal de Nível Superior (CAPES), pelo apoio financeiro ao desenvolvimento da pesquisa.

\section{REFERÊNCIAS}

Agência Nacional de Vigilância Sanitária - ANVISA. (2005, setembro 22). RDC n 263, de 22 de Setembro/2005. Diário Oficial [da] República Federativa do Brasil, Brasília. Recuperado em 06 fevereiro,2019, de http://portal.anvisa.gov.br/documents/10181/2718376/RDC_263_2005_.pdf/2b494d48-0d39-4c8d-84d1-e20ec6e9190f

Almeida, T. M., Santos, M. R. L., \& Belo, E. S. (2017, Setembro 25-28). Análise Físico-química de Farinha de Banana Verde. VI Congresso Estadual de Iniciação Científica e Tecnológica do IF Goiano, Goiânia, Goiás. Recuperado em 06 fevereiro,2019, de https://even3storage.blob.core.windows.net/anais/62186.pdf

Andrade, B. A., Perius, D. B., Mattos, N. V. D., Luvielmo, M. D. M., \& Mellado, M. S. (2018). Produção de farinha de banana verde (Musa spp.) para aplicação em pão de trigo integral. Brazilian Journal of Food Technology, 21, 2-10.

Asmar, S. A., Castro, E. M., Pasqual, M., Pereira, F. J., \& Soares, J. D. R. (2013). Changes in leaf anatomy and photosynthesis of micropropagated banana plantlets under different silicon sources. Scientia Horticulturae, 161, 328-332. http://dx.doi.org/10.1016/j.scienta.2013.07.021

Bertolini, A. C., Bello-Pérez, L. A., Méndez-Montealvo, G., Almeida, C. A., \& Lajolo, F. (2010). Rheological and functional properties of flours from banana pulp and peel. Stärke, 62(6), 277-284. http://dx.doi.org/10.1002/star.200900216

Bezerra, C. V., Amante, E. R., de Oliveira, D. C., Rodrigues, A. M., \& da Silva, L. H. M. (2013). Green banana (Musa cavendishii) flour obtained in spouted bed-Effect of drying on physico-chemical, functional and morphological characteristics of the starch. Industrial Crops and Products, 41, 241-249. http://dx.doi.org/10.1016/j.indcrop.2012.04.035

Borges, A. D. M., Pereira, J., \& Lucena, E. M. P. D. (2009). Caracterização da farinha de banana verde. Food Science and Technology, 29(2), 333-339. http://dx.doi.org/10.1590/S0101-20612009000200015

Castelo-Branco, V. N., Guimarães, J. N., Souza, L., Guedes, M. R., Silva, P. M., Ferrão, L. L., Miyahira, R. F., Guimarães, R. R., Freitas, S. M. L., Reis, M. C., \& Zago, L. (2017). Uso da farinha de polpa e de casca de banana verde (Musa balbisiana) como ingrediente para a elaboração de massa tipo talharim. Brazilian Journal of Food Technology, 21, 1-8.

Castro, J. M. D. (2019). Produção de cookies isentos de glúten com uso da farinha de banana verde e farinha de arroz (Trabalho de conclusão de curso). Instituto Federal Goiano, Urutaí, GO.

Coelho Junior, L. M. (2013). Concentração regional do valor bruto de produção da banana do Paraná, Brasil (1995 a 2010). Ciência Rural, 43(12), 2304-2310. http://dx.doi.org/10.1590/S0103-84782013005000137

De Melo, J. V. D., Formiga, M. W. M., de Andrade, J. L., Gouveia, L. D. G., Vieira, J. K. B., Gomes, Á. K. G., \& de Sousa, M. N A. (2019). Efeitos dos fitoesteróis para a prevenção de doenças. Revista Brasileira de Educação e Saúde, 9(1), 27-31. 
Food and Agriculture Organization of the United Nations - FAO. (2011). Consumo. Recuperado em 12 dezembro, 2018, de http://www.faostat.fao.org/site/340/default.aspx

Gonçalves, J. Q., da Silva, M. A. P., Plácido, G. R., Caliari, M., Silva, R. M., Moura, L. C., \& Souza, D. G. (2017). Secagem da casca e polpa da banana verde (Musa acuminata): Propriedades físicas e funcionais da farinha. Global Science and Technology, 9(3), 62-72.

Haslinda, W. H., Cheng, L. H., Chong, L. C., \& Aziah, A. N. (2009). Chemical composition and physicochemical properties of green banana (Musa acuminatax balbisiana Colla cv. Awak) flour. International Journal of Food Sciences and Nutrition, 60(Suppl. 4), 232-239.

Instituto Adolfo Lutz - IAL. (2008). Métodos físico-químicos para análise de alimentos: Normas analíticas do Instituto Adolfo Lutz. Recuperado em 20 de abril de 2018, de

http://www.ial.sp.gov.br/resources/editorinplace/ial/2016_3_19/analisedealimentosial_2008.pdf?attach=true

Instituto Brasileiro de Geografia e Estatítica - IBGE. (2017). Levantamento Sistemático da Produção Agrícola. Pesquisa Mensal de Previsão e Acompanhamento das Safras Agrícolas no Ano Civil. Recuperado em 29 de maio de 2018, de https://biblioteca.ibge.gov.br/index.php/biblioteca-catalogo?view=detalhes\&id=76.

Juarez-Garcia, E., Agama-Acevedo, E., Sáyago-Ayerdi, S. G., Rodriguez-Ambriz, S. L., \& Bello-Perez, L. A. (2006). Composition, digestibility and application in breadmaking of banana flour. Plant Foods for Human Nutrition, 61(3), 131-137. PMid:17048100. http://dx.doi.org/10.1007/s11130-006-0020-x

Leonel, M., Carmo, E. L. D., Leonel, S., Franco, C. M. L., \& Campanha, R. B. (2011). Extração e caracterização do amido de diferentes genótipos de bananeira. Revista Brasileira de Fruticultura, 33(Spe 1), 599-605.

Matsuura, F. C. A. U., Costa, J. I. P. D., \& Folegatti, M. I. D. S. (2004). Marketing de banana: preferências do consumidor quanto aos atributos de qualidade dos frutos. Revista Brasileira de Fruticultura, 26(1), 48-52. http://dx.doi.org/10.1590/S010029452004000100014

Mattsson, L., Williams, H., \& Berghel, J. (2018). Waste of fresh fruit and vegetables at retailers in Sweden-Measuring and calculation of mass, economic cost and climate impact. Resources, Conservation and Recycling, 130, 118-126. http://dx.doi.org/10.1016/j.resconrec.2017.10.037

Melo, P. R. L. (2014). Leite fermentado probiótico com adição de farinha de banana verde (Dissertação de mestrado). Centro Universitário do Instituto Mauá de Tecnologia, São Caetano do Sul, SP.

Mendes-Filho, N. E., Carvalho, M. P., \& Souza, J. M. T. (2014). Determinação de macrocomponentes e nutrientes minerais da polpa de manga (Mangifera indica L.). Revista Eletrônica Perspectivas da Ciência e Tecnologia, 6(1-2), 22.

Menezes, C. C., Borges, S. V., Cirillo, M. A., Ferrua, F. Q., Oliveira, L. F., Mesquita, K. S. (2009). Caracterização física e físicoquímica de diferentes formulações de doce de goiaba (Psidiumguajava L.) da cultivar Pedro Sato. Ciência e Tecnologia de Alimentos, 29(3),618-625.

Mota, R. V., Lajolo, F. M., Cordenunsi, B. R., \& Ciacco, C. (2000). Composition and functional properties of banana flour from different varieties. Stärke, 52(2-3), 63-68. http://dx.doi.org/10.1002/(SICI)1521-379X(200004)52:2/3<63::AIDSTAR63>3.0.CO;2-V

Nascimento, L. M. G., Amaral, M. C. A., Santos, M., Ramos, B., Ribeiro, S. D. O., \& Veloso, C. (2017). Farinha de banana da terra verde: caracterização química e propriedades tecnológicas. In VIII Semana de Agronomia: Os desafios para a agricultura no século XXI, Vitória da Conquista, Bahia.

Pires, V. C. F., Silva, F. L. H., \& Souza, R. M. S. (2014). Parâmetros da secagem da banana pacovan e caracterização físicoquímica da farinha de banana verde. Revista Verde de Agroecologia e Desenvolvimento Sustentável, 9(1), 28. http://dx.doi.org/10.18378/rvads.v9i1.2577

Programa Brasileiro para a Modernização da Horticultura - PBMH \& Produção Integrada de Frutas - PIF. (2006). Normas de classificação de banana. São Paulo: CEAGESP. Recuperado em 19 dezembro,2018, de https://pt.scribd.com/document/368500273/BANANA-NORMAS-DE-CLASSIFICACAO-pdf .

Ramos, D. P., Leonel, M., \& Leonel, S. (2010). Amido resistente em farinhas de banana verde. Alimentos e Nutrição Araraquara, 20(3), 479-484.

Sarawong, C., Schoenlechner, R., Sekiguchi, K., Berghofer, E., \& Ng, P. K. (2014). Effect of extrusion cooking on the physicochemical properties, resistant starch, phenolic content and antioxidant capacities of green banana flour. Food Chemistry, 143, 33-39. PMid:24054209. http://dx.doi.org/10.1016/j.foodchem.2013.07.081

Sardá, F. A. H. (2015). Farinha de banana verde: Efeitos fisiológicos do consumo regular sobre a fome/saciedade e microbiota intestinal em voluntários saudáveis (Tese de doutorado). Universidade de São Paulo, São Paulo.

Savlak, N., Türker, B., \& Yeşilkanat, N. (2016). Effects of particle size distribution on some physical, chemical and functional properties of unripe banana flour. Food Chemistry, 213, 180-186. PMid:27451170. http://dx.doi.org/10.1016/j.foodchem.2016.06.064

Secretaria de Agricultura e do Abastecimento. (2016). Análise da Conjuntura Agropecuária SAFRA 2016/17. Recuperado em 11 março de 2019, de http://www.agricultura.pr.gov.br/arquivos/File/deral/Prognosticos/2017/Fruticultura_2016_17.pdf

Souza, M. E. D., Leonel, S., \& Fragoso, A. M. (2011). Crescimento e produção de genótipos de bananeiras em clima subtropical. Ciência Rural, 41(4), 587-591. http://dx.doi.org/10.1590/S0103-84782011005000032

Tribess, T. B., Hernández-Uribe, J. P., Méndez-Montealvo, M. G. C., Menezes, E. W. D., Bello-Perez, L. A., \& Tadini, C. C. (2009). Thermal properties and resistant starch content of green banana flour (Musa cavendishii) produced at different drying conditions. Lebensmittel-Wissenschaft + Technologie, 42(5), 1022-1025. http://dx.doi.org/10.1016/j.Iwt.2008.12.017 
Vernaza, M. G., Gularte, M. A., \& Chang, Y. K. (2011). Addition of green banana flour to instant noodles: rheological and technological properties. Ciência e Agrotecnologia, 35(6), 1157-1165. http://dx.doi.org/10.1590/S1413-70542011000600016

Wang, Y., Zhang, M., \& Mujumdar, A. S. (2012). Influence of green banana flour substitution for cassava starch on the nutrition, color, texture and sensory quality in two types of snacks. Lebensmittel-Wissenschaft + Technologie, 47(1), 175-182. http://dx.doi.org/10.1016/j.Iwt.2011.12.011

Waszak, M. N., \& Ferreira, C. C. D. (2011). Efeito hipoglicemiante das farinhas de banana verde e de maracujá no controle da glicemia em diabéticos. Cadernos UniFOA, 6(1), 41-50.

Funding: Conselho Nacional de Desenvolvimento Científico e Tecnológico (CNPq) n¹47854/ 2018-0, Fundação de Amparo a Ciência e Tecnologia de Pernambuco (FACEPE) n APQ-0387-

5.07/18, Coordenação de Aperfeiçoamento de Pessoal de Nível Superior- CAPES (Bolsa de mestrado). 